\title{
Occult Hepatitis B Virus Infection in a Previously Vaccinated Injection Drug User
}

\author{
Eleanor A. Powell, ${ }^{1}$ Sanam Razeghi, ${ }^{1}$ Stephen Zucker, ${ }^{1}$ and Jason T. Blackard ${ }^{1, *}$ \\ ${ }^{1}$ Division of Digestive Disease, Department of Internal Medicine, University of Cincinnati College of Medicine, Cincinnati, OH, USA \\ *Corresponding Author: Jason T. Blackard, Division of Digestive Disease, Department of Internal Medicine, University of Cincinnati College of Medicine, Cincinnati, ML 0595, 231 \\ Albert Sabin Way Cincinnati, OH 45267, USA. Tel: +1-513-558-4389, Fax: +1-513-558-1744, E-mail: jason.blackard@uc.edu \\ Received 2015 November 16; Accepted 2015 December 13.
}

\begin{abstract}
Introduction: Occult hepatitis B virus (HBV) is defined by the presence of HBVDNAin patient sera in the absence of HBsAg. Occult HBVhas been associated with hepatocellular carcinoma, reactivation during immune suppression, and transmission to others. While the hepatitis $B$ vaccine is very effective at preventing chronic $\mathrm{HBV}$ infection, recent studies indicate it is less effective at preventing occult $\mathrm{HBV}$ following infant vaccination. No studies, however, have examined the efficacy of adult HBV vaccination at preventing occult HBV. Here, we present the first report of occult $\mathrm{HBV}$ following adult vaccination.

Case Presentation: A 21-year old Caucasian female presented with tricuspid valve endocarditis secondary to methicillin-susceptible Staphylococcus aureus with non-ischemic cardiomyopathy. She reported active use of intravenous drugs. Her liver enzymes were elevated $($ ALT $=1873 \mathrm{IU} / \mathrm{mL} ; \mathrm{AST}=4518 \mathrm{IU} / \mathrm{mL}$ ), and she was found to have HCV and occult HBV. HBV viral loads ranged from $4608-8364 \mathrm{copies} \mathrm{IU} / \mathrm{mL}$ during hospitalization. The patient's HBV was sequenced and found to be genotype D3 without any known diagnostic escape mutations. Immune complexes that may have prevented HBsAg detection were not observed.

Conclusions: $\mathrm{HBV}$ vaccination in infancy is effective at preventing chronic HBV infection but is less effective at preventing occult HBV infection. Similar studies examining the efficacy of adult $\mathrm{HBV}$ vaccination in preventing occult $\mathrm{HBV}$ have not been performed. This case highlights the importance of carefully determining the HBV status of high-riskindividuals, as vaccination history and the presence of antiHBs may not be adequate to rule out HBV infection, even in the absence of HBsAg.

Keywords: Hepatitis B, Occult Hepatitis B, Hepatitis B Vaccine
\end{abstract}

\section{Introduction}

Despite the availability of an effective vaccine, hepatitis B (HBV) remains a global health problem. Two billion people have been exposed, and approximately 240 million people are living with chronic infection (1). Chronic $\mathrm{HBV}$ is defined by the presence of hepatitis B surface antigen (HBsAg) in patient serum for at least six months, while occult HBV is characterized by undetectable HBsAg in the presence of HBV DNA in patient serum. Several studies have shown that, while infant vaccination provides excellent protection against the development of $\mathrm{HBs} A g$ positive $\mathrm{HBV}$, it is less effective at preventing occult HBV (2-5). The acquisition of occult HBV following neonatal vaccination has been reported to occur both vertically, from HBsAg positive mothers $(2,4,5)$ and horizontally (3). However, studies are lacking in those who have received the HBV vaccine after infancy. Here, we report a case of resolving occult HBV infection resulting in a liver enzyme flare following vaccination as an adult.

\section{Case Presentation}

In November of 2012, a 21-year old caucasian American woman was admitted to the university of Cincinnati medical center with tricuspid valve endocarditis secondary to methicillin-susceptible Staphylococcus aureus which was complicated by non-ischemic cardiomyopathy (ejection fraction 15\%). She reported active use of intravenous drugs. The patient tested positive for antibodies to the hepatitis $\mathrm{C}$ virus (HCV), with initially undetectable $\mathrm{HCV}$ RNA and subsequent low-level viremia (174- $902 \mathrm{IU} / \mathrm{mL}$ during hospitalization). The patient was anti-HBs positive, anti-HBc negative, and HBsAg negative (Table 1) consistent with her reported history of prior vaccination as part of a nursing training program several years prior to her hospital admission. Despite a persistently negative HBsAg, HBeAg was positive, and HBV DNA was detectable in three separate serum samples (Table 1). During the course of the hospitalization, the patient seroconverted to become anti-HBc positive. She received no antiviral medications and, at follow-up six months later, she was found to be HBeAg negative and anti-HBe positive, and no longer had detectable HBV DNA, indicating that her occult HBV infection had resolved.

Copyright ( ) 2016, Kowsar Corp. This is an open-access article distributed under the terms of the Creative Commons Attribution-NonCommercial 4.0 International License (http://creativecommons.org/licenses/by-nc/4.0/) which permits copy and redistribute the material just in noncommercial usages, provided the original work is properly cited. 
To investigate which factors may have contributed to the occult phenotype of the vaccine breakthrough infection, several laboratory analyses were performed. First, the patient's serum was subjected to acid dissociation in order to separate antigen-antibody complexes (6). While both the dissociated and un dissociated samples were negative for HBsAg when utilizing the original clinical HBsAg assay, the dissociated sample was positive for HBsAg via the Biochain ELISA (Newark, CA, USA) (Table 2). Taken together, these data suggest that, while immune complexes were present in the patient serum, sequestration of HBsAg in these complexes does not account for the lack of detectable surface antigen by the clinical HBsAg assay and the occult HBV phenotype.

Next, full-length HBV sequences were obtained using rolling circle amplification followed by PCR (7). Nine full-length $\mathrm{HBV}$ clones were sequenced as previously described (8). Sequence chromatographs were assembled and edited using CodonCode Aligner (CodonCode, Centerville, Massachusetts). Sequences were aligned in ClustalX with full-length GenBank references. The statistical robustness and reliability of these trees was determined using bootstrap analysis with 1,000 replicates. Additional phylogenetic inference was performed using a Bayesian Markov chain Monte Carlo (MCMC) approach as implemented in the Bayesian evolutionary analysis by sampling trees (BEAST) v1.8.0 program (9) under an uncorrelated log-normal relaxed molecular clock and the
Hasegawa, Kishino and Yano (HKY) model with nucleotide site heterogeneity estimated using a gamma distribution. The BEAST MCMC analysis was run for a chain length of $100,000,000$ with sampling every 10,000th generation. Results were visualized in Tracer v1.5 to confirm chain convergence, and the effective sample size (ESS) was calculated for each parameter. All ESS values were $>200$ indicating sufficient sampling. The maximum clade credibility tree was selected from the posterior tree distribution after a 10\% burn-in using TreeAnnotator v1.8.0. Posterior probabilities $>90 \%$ were considered statistically significant.

All nine of the HBV clones from the patient were found to be genotype D3 (Figure 1). Genetic distances were calculated by pairwise comparison of nucleotide sequences using the Kimura method of MegAlign (DNASTAR, Madison, WI). The mean genetic distance between samples was $0.29 \%$, with a range of $0.12 \%$ $0.47 \%$, indicating that all the intrapatient viral variants are closely related, and this may be indicative of recent infection. Examination of the "a" determinant, the region of HBsAg against which antibodies react, revealed no mutations associated with diagnostic failure or vaccine escape (10). As such, viral mutations are unlikely to play a role in either the ability of HBV to infect despite vaccination. The lack of surface antigen is also unlikely to be caused by failure of the diagnostic assay to detect HBsAg.

\begin{tabular}{|c|c|c|c|c|c|c|c|c|}
\hline & $11 / 19 / 13$ & $1 / 17 / 14$ & 1/18/14 & 1/24/14 & $1 / 25 / 14$ & 1/29/14 & 1/30/14 & 7/9/14 \\
\hline ALT, IU/mL & & 1873 & & 561 & & & & \\
\hline AST, IU/mL & & 4518 & & 203 & & & & \\
\hline Anti-HCV & Positive & & & & & & & \\
\hline HCV viral load, IU/mL & Undetectable & 902 & 642 & & 174 & & & Undetectable \\
\hline HBsAg & & Negative & & & Negative & & & Negative \\
\hline Anti-HBs & Positive & & & & Positive & Positive & & Positive \\
\hline Anti-HBc total & Negative & & & & Positive & & Positive & Positive \\
\hline Anti-HBc IgM & & & & & & & Negative & \\
\hline HBeAg & & & & & Positive & Positive & & Negative \\
\hline Anti-HBe & & & & & Negative & Negative & & Positive \\
\hline HBV viral load, IU/mL & & & 8364 & & 4608 & 5243 & & Undetectable \\
\hline Anti-HIV & Negative & & & & & & & \\
\hline
\end{tabular}

Table 2. Acid Dissociation of Immune Complexes in Patient Seruma

\begin{tabular}{|c|c|c|}
\hline & Clinical HBsAg Assay & Research HBsAg ELISA \\
\hline Patient serum before acid dissociation & Negative (0.09 arbitrary units) & Negative $(\mathrm{OD}=0.085)$ \\
\hline Patient serum following acid dissociation & Negative (0.41 arbitrary units) & Positive $(\mathrm{OD}=0.247)$ \\
\hline
\end{tabular}

${ }^{a}$ One hundred microliters of patient serum was treated as described previously (6) and untreated serum was diluted with phosphate buffered saline to account for the volume changes due to treatment. Both dissociated and diluted, untreated samples were tested using the clinical HBsAg assay, and HBsAg positivity was determined via assay guidelines ( 0 - 0.99 arbitrary units is negative). Samples were also evaluated by a research HBsAg ELISA; the sample was considered positive if the optical density (OD) value was greater than 2.1 times the value of the assay negative control $(\mathrm{OD}=0.103)$. 


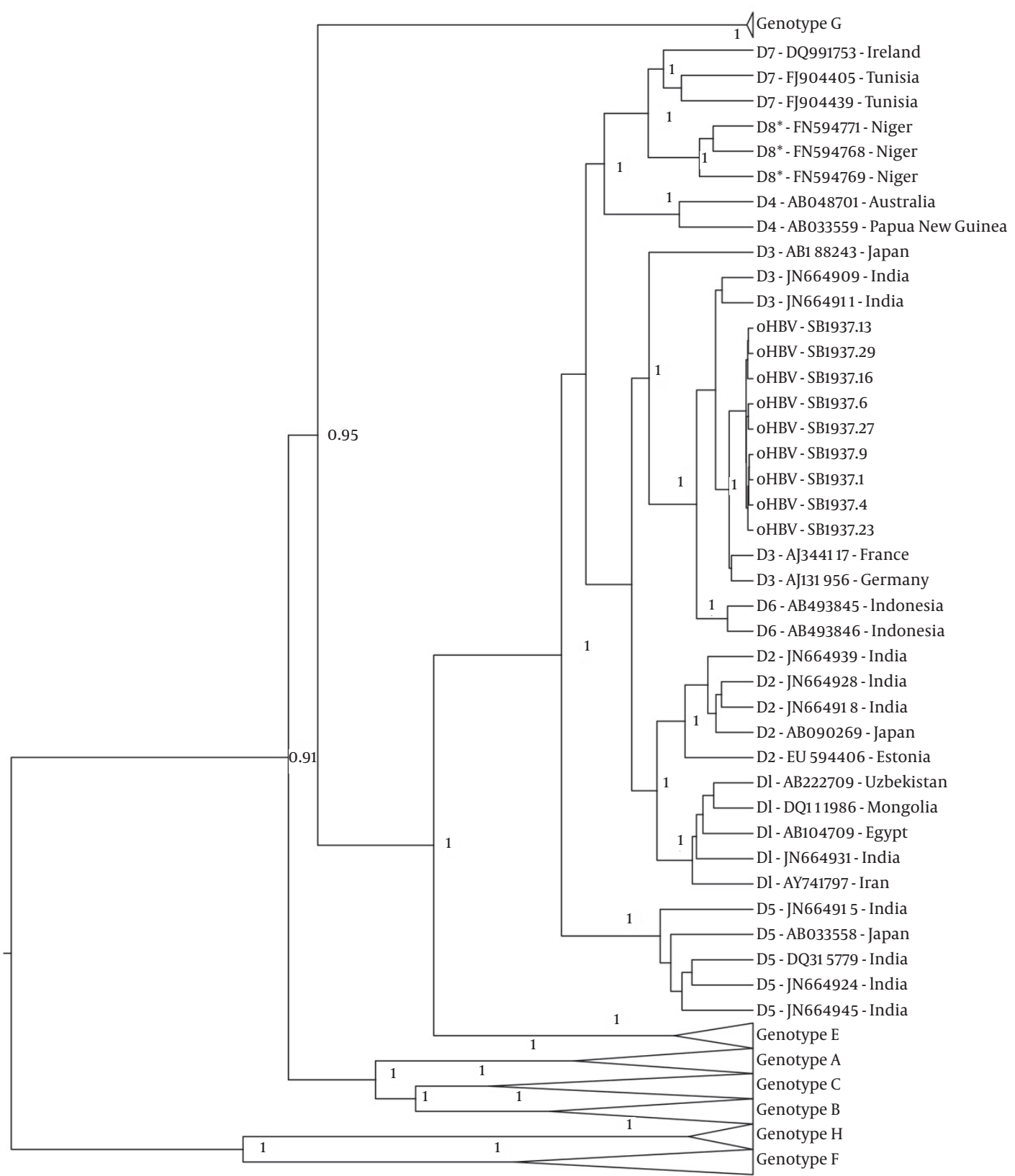

0.02

Three references of each genotype or subgenotype were aligned with patient samples. Bayesian inference was utilized to analyze the relatedness of samples. Patient sequences are labeled "SB1937.X" where X is the viral clone number. References are labeled "Y-accession number" where Y is the genotype of the reference. Relevant posterior probabilities greater than 0.9 are shown.

\section{Discussion}

A growing body of evidence suggests that hepatitis B vaccination is very effective at preventing HBsAg positive HBV infection but is less so for HBsAg negative (occult) HBV. One study followed HBs-vaccinated infants born to HBsAg mothers in China and reported that $4.92 \%$ developed occult HBV compared to $1.61 \%$ who developed chronic HBV (5). A similar study from India found that, at 18 weeks post-partum, $64 \%$ of vaccinated babies born to HBsAg positive mothers had developed occult HBV, while only $3 \%$ were HBsAg positive (2). At twenty-four 
months of age, $42 \%$ of the vaccinated infants had persistent occult HBV, while $27 \%$ had lost HBV DNA and developed adequate immune response. Finally, a report from Iran found that $28 \%$ of children born to HBsAg-positive mothers who were positive for anti-HBs following vaccination went on to develop occult HBV (4). Several studies examine occult HBV on a population level following implementation of infant vaccination programs. A Taiwanese study found that universal infant vaccination reduced the calculated prevalence of occult HBV among children from $16.1 \%$ in unvaccinated cohorts to $1.2 \%$ in vaccinated cohorts (11). A South African study found that the prevalence of occult $\mathrm{HBV}$ in anti-HBc positive individuals was reduced from $70.4 \%$ prior to the vaccination program to $66.0 \%$ in the post-vaccination era (12). One long-term follow-up study reported that, of 2919 Chinese young adults vaccinated as infants, $2.1 \% \mathrm{mani}$ fested chronic HBV, while $4.2 \%$ had occult HBV at age 19 - 21 (13). In contrast with vertical transmission, studies of horizontal transmission of HBV following successful vaccination as an adult are limited, although HBsAgpositive vaccine escape infections have been reported (13). Two cases of resolving acute occult HBV have been previously described; in both instances these were asymptomatic infections without elevated liver enzymes in unvaccinated individuals $(14,15)$.

In the present case, we report resolving occult $\mathrm{HBV}$ in an HCV-positive IDU following vaccination. To our knowledge, this is the first case of occult HBV reported following adult vaccination. HBV DNA was detected in the patient's serum in the setting of an acute rise in liver enzymes. While there are a number of factors that may have contributed to the patient's liver injury (medications, heart failure), these findings suggesting that occult HBV may be detrimental to the liver. This case highlights the possible presence of viremia in high-risk individuals, even in the setting of detectable anti-HBs and the absence of HBsAg.

The mechanism by which viremia occurred in the absence of HBsAg is unclear, as none of the viral clones exhibited known vaccine-escape mutations. Furthermore, no diagnostic failure mutations were found in any of the viral clones from this patient, and dissociation of immune complexes led to only a small increase in free HBsAg. Even after dissociation, the clinical HBsAg assay was unable to detect HBsAg, suggesting sequestration of HBsAg in immune complexes does not account for the occult phenotype of this infection.

This case report has several potential limitations. First, it consists only of one patient and will need to be validated in a larger study population. This case does, however, highlight the need for studies of the prevalence of occult

\section{References}

1. World Health Organization. WHO | Hepatitis B [Internet]. Fact Sheet N204: Hepatitis B. World Health Organization; 2013. Available from: http://www.who.int/mediacentre/factsheets/fs204/en/.

2. Pande C, Sarin SK, Patra S, Kumar A, Mishra S, Srivastava S, et al.
$\mathrm{HBV}$ in individuals who were vaccinated as adults. Unfortunately, the patient was not tested for anti-HBc IgM until weeks after her presentation, so it is unclear whether the patient had recently acquired $\mathrm{HBV}$. We are unable to exclude the possibility that the patient was infected before vaccination and had sustained viral replication at a very low level. This is unlikely, however, as the patient was previously both anti-HBc and anti-HBe negative, suggesting the only viral protein the patient was exposed to was HBsAg, indicating a history of vaccination. Given the limited number of viral clones that were sequenced, there is the potential that minor mutations that contribute to the observed phenotype were overlooked. While we have previously demonstrated that sequencing ten clones is adequate to capture much of the quasi species diversity in HBV infection (16), it is possible that minor mutations could exert a dominant negative effect over wild-type viruses with regard to HBsAg production.

In summary, here we report a case of resolving occult HBV following adult vaccination in an HCV-positive IDU. Previous studies have indicated that while HBs vaccination is effective at preventing chronic HBV infection, it may be less effective at preventing HBsAg-negative HBV infection. This case highlights the importance of carefully determining the HBV status of high-risk individuals, as vaccination history and the presence of anti-HBs may not be adequate to rule out $\mathrm{HBV}$ infection, even in the absence of HBsAg.

\section{Acknowledgments}

This study was approved by the institutional review board at the University of Cincinnati College of Medicine on January 14, 2014. The subject provided written informed consent for participation in this study at time of sample collection. A copy of the written consent is available for review by the Editor of this journal.

\section{Footnotes}

Authors' Contribution:Eleanor A. Powell performed all laboratory experiments, did sequence analysis, and drafted the manuscript. Sanam Razeghi saw the patient and contributed to the overall understanding of the case. Stephen Zucker saw the patient, identified the case as a case of interest, and contributed to the overall understanding of the case. Jason T. Blackard conceived of the study and participated in its design. All authors read and approved of the final manuscript.

Funding/Support:This work was supported in part by NIH award R21 AI096958 to JTB. 
tis. 2013;20(11):801-10.

3. Xu L, Wei Y, Chen T, Lu J, Zhu C, Ni Z, et al. Occult HBV infection in anti-HBs-positive young adults after neonatal $\mathrm{HB}$ vaccination. Vaccine. 2010;28(37):5986-92. [PubMed:20637763]

4. Shahmoradi S, Yahyapour Y, Mahmoodi M, Alavian SM, Fazeli Z, Jazayeri SM. High prevalence of occult hepatitis B virus infection in children born to HBsAg-positive mothers despite prophylaxis with hepatitis B vaccination and HBIG. Int J Hepatol. 2012;57(3):515-21.

5. Su H, Zhang Y, Xu D, Wang B, Zhang L, Li D, et al. Occult hepatitis $B$ virus infection in anti-HBs-positive infants born to HBsAg-positive mothers in China. PLoS One. 2013;8(8):e70768.

6. Weber B, Melchior W, Gehrke R, Doerr HW, Berger A, Rabenau H. Hepatitis B virus markers in anti-HBc only positive individuals. Med Virol. 2001;64(3):312-9. [PubMed:11424120]

7. Martel N, Gomes SA, Chemin I, Trépo C, Kay A. Improved rolling circle amplification (RCA) of hepatitis B virus (HBV) relaxedcircular serum DNA (RC-DNA). J Virol Methods. 2013;193(2):653-9. [PubMed: 23928222]

8. Gunther S, Li BC, Miska S, Kruger DH, Meisel H, Will H. A novel method for efficient amplification of whole hepatitis B virus genomes permits rapid functional analysis and reveals deletion mutants in immunosuppressed patients. JVirol.1995;69(9):543744. [PubMed:7636989]

9. Drummond AJ, Rambaut A. BEAST: Bayesian evolutionary analysis by sampling trees. BMC Evol Biol. 2007;7(1):214. [PubMed: 17996036]

10. Vivekanandan P, Kannangai R, Ray SC, Thomas DL, Torbenson $\mathrm{M}$. Comprehensive genetic and epigenetic analysis of oc- cult hepatitis B from liver tissue samples. Arch Clin Infect Dis. 2008;46(8):1227-36.

11. Hsu HY, Chang MH, Ni YH, Chiang CL, Wu JF, Chen HL. Universal infant immunization and occult hepatitis $B$ virus infection in children and adolescents: a population-based study. Hepatology. 2015;61(4):1183-91. doi: 10.1002/hep.27650. [PubMed: 25501911]

12. Amponsah-Dacosta E, Lebelo RL, Rakgole JN, Selabe SG, Gededzha MP, Mayaphi SH, et al. Hepatitis B virus infection in postvaccination South Africa: Occult HBV infection and circulating surface gene variants. J Clin Virol. 2015;63:12-7. doi: 10.1016/j. jcv.2014.11.032. [PubMed: 25600597]

13. van Dommelen L, Verbon A, van Doorn HR, Goossens VJ. Acute hepatitis B virus infection with simultaneous high HBsAg and high anti-HBs signals in a previously HBV vaccinated HIV-1 positive patient. J Clin Virol. 2010;47(3):293-6. [PubMed: 20060779]

14. Manzini P, Abate ML, Valpreda C, Milanesi P, Curti F, Rizzetto M, et al. Evidence of acute primary occult hepatitis B virus infection in an Italian repeat blood donor. Transfusion. 2009;49(4):757-64. [PubMed: 19171000]

15. Weber B, Muhlbacher A, Melchior W. Detection of an acute asymptomatic HBsAg negative hepatitis $B$ virus infection in a blood donor by HBV DNA testing. J Clin Virol. 2005;32(1):67-70. [PubMed:15572009]

16. Martin CM, Welge JA, Shire NJ, Rouster SD, Shata MT, Sherman $\mathrm{KE}$, et al. Genomic variability associated with the presence of occult hepatitis B virus in HIV co-infected individuals. J Viral Hepat. 2010;17(8):588-97. doi: 10.1111/j.1365-2893.2009.01214.x. [PubMed: 19889143] 